\title{
ACUTE PAINLESS VISUAL LOSS: WHAT THE EYES CANNOT SEE, THE MIND SHOULD KNOW'
}

\author{
Sandip Sahu ${ }^{1}$, Sayali Mishra ${ }^{2}$, Biswa Ranjan Mishra ${ }^{2}$, Santanu Nath $^{3}$ \& Debadatta Mohapatra ${ }^{2}$ \\ ${ }^{1}$ Department of Opthalmology, All India Institute of Medical Sciences (AIIMS), Bhubaneswar, India \\ ${ }^{2}$ Department of Psychiatry, All India Institute of Medical Sciences (AIIMS), Bhubaneswar, India \\ ${ }^{3}$ Department of Psychiatry, All India Institute of Medical Sciences (AIIMS), Deoghar, Jharkhand, India
}

received: 14.4.2021;

revised: 26.6.2021;

accepted: 5.4 .2021

\section{INTRODUCTION}

Visual impairment, either acute or gradual, painful or painless, can range from mild, moderate, severe impairment to blindness which can be challenging to adapt for an individual. The prevalence of blindness in India across all age groups is $0.36 \%$, with the common causes being cataract, uncorrected refractive disorders, corneal opacity and glaucoma (Das 2018). The impairment of vision despite normal ophthalmological findings, is usually accompanied by neurological abnormalities, localizing the deficit in the visual cortex. In emergency setting, acute painless visual impairment without any definable ophthalmological or central nervous system findings can be quite challenging for an ophthalmologist (Mulugeta et al. 2015).

We are hereby reporting a case of acute, painless loss of vision showing dramatic recovery with psychological intervention, emphasizing the need to consider the role of psychological factors in such scenario.

\section{CASE PRESENTATION}

A 13-years-old female presented with a 5 days history of abrupt onset of gross diminution of vision in her right eye, which was associated with infrequent peribulbar pain for the initial 2 days. However, during this period she could make out light sources but could not see the objects and people in the right visual field. There was no diplopia, tunnel vision, hemianopia, headache, vomiting, and neither any history of trauma, diabetes, hypertension, seizures, or vasculitis. Ophthalmological examination revealed her vision in right eye to be 20/200 and left eye $20 / 20$. The cornea was clear and the anterior chamber was within normal limits, with no relative afferent pupillary defect and there was full range of extra-ocular movements. On fundoscopy, there was no evidence of papilledema. The patient was provisionally diagnosed as right retrobulbar neuritis. In order to rule out any central cause, Visual evoked potential study and MRI brain were done, but found out to be normal. Hence, to rule out any psychogenic basis for the presentation, a psychiatric consultation was sought.

Her mental status examination revealed a cooperative attitude, average psychomotor activity, speaking in a childish manner, euthymic affect and interestingly with- out any overt distress regarding her sudden mono-ocular blindness. In view of the dramatic presentation without any evident organicity and "la bella indifference" in affect, the possibility of Dissociative disorder (sensory) was considered. In order to explore the psychological conflict, Lorazepam assisted interview was done which revealed that a day before, she evidenced her friend being punished by the class teacher which was painful to see and a surprise examination was announced the day after for which she was unprepared. Due to the current presentation, her parents had to rush to the hospital and she missed the exam for that day. Immediately after revealing the conflict, there was dramatic return of sight and she was explained the link between the nature of psychological conflict and her symptoms, with some positive suggestions. The parents were explained about the psychological nature of the symptoms, advised to cut down secondary gains and encourage her going to school.

\section{DISCUSSION}

Sudden onset of visual loss without any identifiable ocular or central cause accounts for about $1 \%$ of the presentations in emergency ophthalmology settings (Beatty 1999). Functional visual loss encompasses terminologies like 'hysterical' (amblyopia), 'conversion disorder' etc and are more common in adolescents, young adults and females. They present with acute onset of visual diminution/loss associated with or without visual field deficits (36-80\%). The visual symptoms involve both the eyes in the majority (50-80\%) and isolated field deficits are rare (Sletteberg et al. 1989).

In our case, there was acute and dramatic loss of mono-ocular vision without any evidence of organicity in ophthalmological and neuroradiological examinations. Interestingly, they can have an intact menace reflex and flinching with increased illumination. The complaints of loss of visual acuity can be tested by certain techniques like fogging test, prism shift test, mirror test etc., by which the consistency of the complaints can be ascertained (Beatty 1999). Non-organic loss of visual field may occur in association with complaints of impairment in visual acuity. The concentric loss of peripheral vision with consequential 'tunnel vision' is the commonest psychogenic field defects described. 
Table 1. Criteria for the diagnosis of a psychogenic blindness

\begin{tabular}{ll}
\hline \multicolumn{1}{c}{ History } & \multicolumn{1}{c}{ Physical Examination } \\
\hline $\begin{array}{l}\text { Abrupt onset (maximal symptoms at that time) } \\
\text { Fluctuating course }\end{array}$ & $\begin{array}{l}\text { Inconsistent symptoms } \\
\text { Variability over time (frequency, amplitude, } \\
\text { direction/distribution of diminution of vision) }\end{array}$ \\
$\begin{array}{l}\text { Spontaneous remissions/ cures } \\
\text { Distractibility reduces or resolves, attention alters the severity } \\
\text { Paroxysmal symptoms }\end{array}$ & Selective disability \\
Psychiatric comorbidities & Severity inconsistent with the organicity \\
Secondary gain (often not apparent) & Suggestibility \\
Risk factors for dissociative disorder & Functional disability out of proportion to examination findings \\
Psychological stressors(immediate) & La belle Indifference \\
Multiple somatizations/undiagnosed & \\
conditions in the past & \\
\hline
\end{tabular}

However, appropriate techniques like tangent screen testing, Goldmann perimetry and plotting of the visual field with both eyes open are employed to ascertain the genuineness of the complaints (Beatty 1999, Kathol et al. 1983). In absence of any identifiable organic etiology for the acute blindness and then corroborating by the appropriate techniques, the psychogenic factors behind the presentation needs to be understood (Table 1).

Psychogenic visual impairment can be dissociative, factitious or malingering in nature. Individuals with dissociative blindness are suggestible without any apparent distress because of the symptoms, whereas malingers try to convince others about their nature of symptoms and the associated difficulties. In factitious blindness or malingering, the person consciously feigns visual loss for secondary gain like getting medical attention or any tangible benefit. In dissociative blindness as in our case, there is usually an unresolved, psychological conflict which is repressed in the unconscious mind. The symptom expression may have a resemblance with the nature of conflict. In our case, the sight of brutal punishment to friend and the surprise exam, produced the unconscious anxiety, which was repressed and hence manifested as dramatic diminution of vision. In dissociative disorders, the individual usually gets temporary relief from psychic anxiety by repressing, thus dissociating from the conflict and secondary gains in terms of attention from others. The surfacing of the conflict into the conscious mind, facilitated through direct or chemical assisted interview, followed by positive suggestion, leads to dramatic resolution of the symptoms, as evidenced in our case (Kathol et al. 1983).

The case depicted above describes acute visual impairment as an expression of underlying unconscious psychological processes, which should be kept as a possibility in advent of any obvious organic factors. Timely psychological intervention can facilitate a dramatic response, at the same time reducing the treatment costs.

\section{Acknowledgements: None.}

\section{Conflict of interest: None to declare.}

\section{Contribution of individual authors:}

Sandip Sahu: primary treating consultant of patient, diagnosis and management, wrting draft and finalize.

Sayali Mishra: referral treating consultant of patient, diagnosis and management, wrting draft and finalize.

Biswa Ranjan Mishra \& Santanu Nath: diagnosis and management, wrting draft and finalize, proof reading.

Debadatta Mohapatra: diagnosis and management, wrting draft and finalize, proof reading, correction and submission.

\section{References}

1. Beatty S. Non-organic visual loss. Postgrad Med J. 1999;75:201-207. doi:10.1136/pgmj.75.882.201

2. Das T. Blindness and Visual Impairment Profile and Rapid Assessment of Avoidable Blindness in South East Asia: Analysis of New Data. 2017 APAO Holmes Lecture. Asia Pac J Ophthalmol (Phila). 2018;7:312-315. doi:10.22608/APO.2017425

3. Kathol RG, Cox TA, Corbett JJ, Thompson HS, Clancy J. Functional visual loss: I. A true psychiatric disorder?. Psychol Med 1983; 13:307-314. doi:10.1017/s00332917000509232

4. Mulugeta S, Tesfay K, Frank R, Gruber-Frank C. Acute loss of vision in a young woman: a case report on psychogenic blindness. Ethiop J Health Sci 2015; 25:99-104. doi:10.4314/ejhs.v25i1.13

5. Sletteberg $O$, Bertelsen T, Hovding G. The prognosis of patients with hysterical visual impairment. Acta Ophthalmol 1989; 67:159-63

\section{Correspondence:}

Assistant Professor Debadatta Mohapatra, MD

Department of Psychiatry, All India Institute of Medical Sciences (AIIMS)

Flat no 202, B Block, Palm Heights, Bharatpur, Bhubaneswar, 751003, India

E-mail:2000debee@gmail.com 